\title{
ORIGINAL
}

\section{PROFESIONALES DE ATENCIÓN PRIMARIA DE MADRID Y VIOLENCIA DE PAREJA HACIA LA MUJER EN EL AÑ̃ 2010}

Ana Belén Arredondo-Provecho (1), Manuel Broco-Barredo (2), Teresa Alcalá-Ponce de León (3), Araceli Rivera-Álvarez (4), Isabel Jiménez Trujillo (5), Carmen Gallardo-Pino (5).

(1) Servicio de Medicina Preventiva. Hospital Universitario Fundación Alcorcón.

(2) Centro de Salud de Navas del Rey.

(3) Centro de salud "Dos de Mayo" de Móstoles.

(4) Gerencia de Atención Primaria de Móstoles

(5) Medicina Preventiva y Salud Pública. Facultad de Ciencias de la Salud. Universidad Rey Juan Carlos.

\section{RESUMEN}

Fundamentos: La morbilidad asociada a la violencia de pareja hacia la mujer (VPHM) justifica que sus víctimas acudan reiteradamente a los centros de salud. La accesibilidad y continuidad asistencial hacen de la atención primaria (AP) el lugar idóneo para su detección y primer abordaje. Sin embargo, los datos reflejan las dificultades de los/as profesionales para lograr este fin. El objetivo es analizar el nivel de conocimientos, opiniones, barreras organizativas percibidas y propuestas de mejora de los profesionales de AP.

Método: Estudio transversal descriptivo realizado mediante una encuesta anónima y autoadministrada durante los meses de agosto y septiembre de 2010, dirigida a todos/as profesionales de AP del área 8 de Madrid. Se realizó un análisis descriptivo de variables y la chi $^{2}$ para comparar las respuestas obtenidas.

Resultados: Tasa de respuesta $170(21,4 \%) .118(70,7 \%)$ profesionales creen que este es un problema importante y $154(91,7 \%)$ que habitualmente pasa desapercibido. $91(55,2 \%)$ conocen las obligaciones legales que tienen cuando la detectan. $73(51,8 \%)$ piensan que existen barreras organizativas. Entre ellas: presión asistencial 50(29\%), falta de formación específica $40(23,5 \%)$, desconocimiento del procedimiento a seguir $20(11,8 \%)$ y de las competencias de cada profesional $12(7 \%)$.

Conclusiones: El nivel de conocimientos medio para todas las categorías profesionales estudiadas, excepto para trabajo social que es alto.Los/as profesionales de AP consideran que la VPHM es un problema importante que pasa desapercibido. La mitad de ellos/as conocen las obligaciones legales que conlleva la detección. Existen barreras organizativas y estereotipos.

Palabras clave: Violencia doméstica. Mujeres maltratadas. Violencia contra la mujer. Personal de salud. Atención primaria de salud. Conocimientos, actitudes y práctica en salud.

\section{Correspondencia}

Ana Belén Arredondo Provecho

C/ Timanfaya $\mathrm{n}^{\circ} 26,1^{\circ} \mathrm{A}$

28925 Alcorcón. Madrid

Correo electrónico: abarredondo@fhalcorcon.es.

\section{ABSTRACT}

\section{Workers in Primary Health Care and Partner Violence Against Women}

Background: Morbidity associated to partner violence against women (PVAW) justify these patients repeated visits to Health Services. Primary Care is the ideal place for detectión and first aid, due to its easy accesibility and continuated assistance. Nevertheless, numbers show important difficulties to achieve this goal. Our aim is to find out the level of knowledge, opinions, awareness about organizacional barriers and improvement proposals suggested by the workers of primary care.

Method: Cross-sectional descriptive study using an anonymous and voluntary survey during the months of August and September 2010, targeted to all professionals who perform their work in a Primary Care Area of Madrid. We made a descriptive analysis of variables and used $\mathrm{chi}^{2}$ to compare the answers.

Results: Answer rate is 170 (21.4\%). There are stereotypes regarding battered woman and perpetrador. $118(70.7 \%)$ professionals believe that this is a major problem and $154(91.7 \%)$ that usually goes unnoticed. $91(55.2 \%)$ know their legal commitments. $73(51.8 \%)$ think that there are organizational barriers, among them: the burden of care $50(29 \%)$, lack of specific training $40(23.5)$, lack of knowledge about the procedure to be followed $20(11.8 \%)$ and about the professional responsabilities $12(7 \%)$.

Conclusions: All profesional categoríes showed an average level of knowledge, except for social workers that was high. Primary Care workers think that PVAW is an important issue that usually goes unnoticed. Half of them know the legal commitments o detección. There are organizacional barriers and stereotypes

Key words: Domestic violence. Battered women. Violence against women. Health personnel. Primary health care. Health knowledge, attitudes, practice. 


\section{INTRODUCCIÓN}

La violencia de pareja hacia la mujer (VPHM) representa una de las violaciones de los derechos humanos más difundidas y está presente en todos los países, atravesando indistintamente todas las culturas, clases sociales, edades, niveles de educación, categorías económicas y grupos étnicos. Las dimensiones globales del fenómeno son alarmantes ${ }^{1}$.

La Organización de Naciones Unidas (ONU), en su declaración sobre la eliminación de violencia contra la mujer, la define como todo acto de violencia basado en la pertenencia al sexo femenino que tenga o pueda tener como resultado un daño o sufrimiento físico, sexual o psicológico para la mujer, así como las amenazas de tales actos, la coacción o la privación arbitraria de la libertad, tanto si se producen en la vida pública como en la vida privada ${ }^{2}$. Sus parejas o exparejas son quienes la ejercen de forma más habitual ${ }^{3}$.

Esto constituye una preocupación a nivel mundial, de manera que, la $49^{\mathrm{a}}$ Asamblea Mundial de la Salud, en su resolución WHA 49.25, declaró que la violencia es un importante problema de salud pública en todo el mundo, mostrando una gran preocupación por el espectacular aumento de la incidencia de traumatismos causados intencionalmente en todo el mundo a personas de todas las edades y de ambos sexos, pero especialmente a las mujeres y a los niños, por lo que hace un llamamiento para que se adopten y se apliquen políticas y programas específicos de salud pública y servicios sociales encaminados a prevenir la violencia en la sociedad y mitigar sus efectos ${ }^{4}$.

La Organización Mundial de la Salud (OMS), en su Informe Mundial sobre la Violencia y la Salud, recoge la primera recapitulación de la violencia a escala mundial, señalando que es un azote ubicuo que desgarra el tejido comunitario y amenaza la vida, la salud y la felicidad de todos nosotros. En el prefacio se afirma que allí donde la violencia persiste, la salud corre grave peligro ${ }^{5}$. Constituye un grave problema de derechos humanos y salud pública que afecta a todos los sectores de la sociedad ${ }^{6}$. Para dar prioridad a las investigaciones de salud pública sobre violencia, la OMS lideró el Estudio Multipaís sobre Salud de la Mujer y Violencia Doméstica, que refleja una gran diversidad en los datos sobre prevalencia entre países: el porcentaje de mujeres que había sufrido violencia física a manos de sus parejas oscilaba entre el 13\% del Japón y el $61 \%$ en el entorno provincial de Perú. En cuanto a violencia sexual, entre el 6'6\% de Japón y el 59\% de Etiopía. Así mismo, entre el $21 \%$ de Japón y el $90 \%$ de Tanzania se cifraron los porcentajes de mujeres que declararon haber sufrido uno o más comportamientos de maltrato psíquico ${ }^{6}$.

En España, la III macroencuesta de Violencia contra las Mujeres ${ }^{7}$, señala que el 3,6\% de las que residen en España, de 18 o más años, declara haber sido víctima de malos tratos durante el último año por alguna de las personas que conviven en su hogar o por su novio. El 9,6\% de las mujeres residentes en España de 18 o más años son conocidas "técnicamente" como maltratadas. Dentro de este grupo, en el $74,6 \%$ de ellas los actos de violencia eran causados por su pareja. En concreto, en la Comunidad de Madrid la prevalencia fue del 9,4\%. Estos datos de prevalencia reflejan la magnitud del problema, pero la mortalidad que ocasiona resulta mucho más alarmante: el año 2010 acabó con 73 mujeres muertas a manos de su pareja o expareja, de ellas sólo 22 (30\%) habían denunciado, según los datos del Consejo General del poder judicial ${ }^{8}$. 
Recientemente, el Observatorio contra la Violencia Doméstica y de Género, publicó diversas estadísticas basadas en registros reales de la actividad judicial realizada entre julio de 2005 y junio de 2010 , en concreto se contabilizaron 470.706 denuncias en esta materia entre el primer trimestre del 2007 y el segundo trimestre de $2010^{9}$.

Por otra parte, según el Barómetro Sanitario del Centro de Investigaciones Sociológicas del año $2009^{10}$, el grado de confianza que tiene la población respecto a la labor de los/as profesionales que prestan sus servicios en el sistema sanitario es del $84,4 \%$ para los médicos/as y $84,3 \%$ para enfermeras/os. La Institución Sanitaria es altamente valorada por los/as ciudadanos/as en cuanto a la confianza que pueden depositar en ella. Los estudios sobre demandas de asistencia así lo indican, la mayoría de las mujeres que padecen algún tipo de violencia acuden asiduamente a los servicios sanitarios con demandas de distinto tipo ${ }^{10}$.

Por ello, los/as profesionales de la salud, en concreto de Atención Primaria (AP), son un valioso recurso para la detección y el abordaje de la VPHM, tal y como se recoge en la "Guía de Apoyo en Atención Primaria para abordar la violencia de pareja hacia la mujer". Señala que a pesar de la alta prevalencia de la violencia de pareja hacia las mujeres, se detecta poco y suele ser en fases tardías. Las mujeres víctimas de malos tratos acuden durante años a los Centros de Salud antes de reconocer y consultar por violencia ${ }^{11}$. Algunos estudios también ponen de manifiesto una percepción insuficiente desde los servicios sanitarios como problema de salud ${ }^{12}$.

Estas mujeres en comparación con las demás que acuden al centro de salud, muestran una mayor prevalencia en enfermedades crónicas y han debido guardar cama durante más días los 12 meses pre$\operatorname{vios}^{13}$. Los estudios revisados tanto a nivel nacional como internacional en muestras de mujeres que acuden a AP, desvelan una prevalencia de VPHM que oscila entre el 5,5 y el $28,6 \%$ en el último año o anterior y entre el 32 y el $66 \%$ alguna vez en la vida, de las cuales, el $52 \%$ la han sufrido por más de 5 años ${ }^{14-18}$.

Desde el conocimiento de esta alta prevalencia, el papel clave asignado por estas mujeres a los/as profesionales de AP y la labor tan importante que tienen en cuanto a la detección y abordaje de este problema, hacen que su capacitación resulta fundamental.

El objetivo del trabajo fue averiguar el nivel de conocimientos, opiniones, propuestas de mejora para un abordaje integral de los/as profesionales de atención primaria, así como conocer las barreras organizativas que perciben en su labor asistencial habitual.

\section{SUJETOS Y METODOS}

Durante los meses de agosto y septiembre del año 2010 se realizó un estudio transversal descriptivo mediante encuesta anónima autoadministrada para la valoración de los objetivos propuestos en el área 8 de la Comunidad de Madrid. La encuesta se seleccionó a partir de la revisión bibliográfica, tomando como modelo un cuestionario validado mediante su uso en un estudio de similares características realizado por profesionales del Hospital Comarcal Costa del Sol de Marbella en el año $2001^{19}$. Se modificó para adaptarla a las peculiaridades de AP. Se distribuyó a todo el equipo multidisciplinar, considerando que cualquier profesional, tanto sanitario como no sanitario, que desempeña su labor profesional en AP puede ser un referente en la atención a las mujeres que padecen VPHM. También se modifi- 
có el término de la encuesta original: "Violencia Doméstica" por "Violencia de Pareja hacia las Mujeres". Se incluyó el hecho de haber recibido formación específica. Esta misma encuesta se utilizó en otro estudio similar realizado por nosotros/as en los dos hospitales públicos que corresponden a esta misma área de salud, en el año $2008^{20}$.

Se distribuyó en los Centros de Salud, Consultorios Locales y Servicios de Asistencia Rural de Alcorcón, Móstoles, Navalcarnero, San Martín de Valdeiglesias, Villaviciosa de Odón, Villa del Prado, Cadalso de los Vidrios y Navas del Rey. Colaboraron en la difusión y recogida de encuestas: la Gerencia de Área, técnicos/as de Salud, responsables de enfermería y referentes de violencia de los equipos. El área está situada en el suroeste de la Comunidad y da cobertura a una población de 478.082 habitantes, de los cuales 410.456 son mayores de 15 años.

La población diana la formaron un total de 793 profesionales distribuidos por categorías del siguiente modo: 298 médicas/os, 277 enfermeras/os, 29 técnicas/os en cuidados auxiliares de enfermería (TCAE), 42 celadoras/es y 147 auxiliares administrativas/os.

La encuesta constaba de 26 ítems (anexo 1) más los datos sociodemográficos: edad, sexo, estado civil, categoría profesional, centro de trabajo y si se había recibido algún tipo de formación acerca de la VPHM. Valoraba 3 dominios:

A- Nivel de conocimientos (preguntas 1 , $3,4,4.1,5,6,7,8,9,10,11,14,15$ y 16). Se calculó el porcentaje global de aciertos por categoría profesional.

B- Pautas de actuación (preguntas $18 \mathrm{y}$ 19), opiniones (preguntas 12,13, 17, 20, 21 y 22), propuestas de mejora (preguntas 23 y 24) y número de casos vistos en su jornada laboral (pregunta 2).
C- Barreras organizativas y posibles soluciones (preguntas 25 y 26).

Se utilizaron preguntas cerradas de respuesta única a elegir entre dos o más opciones y preguntas abiertas.

La información se recogió en una base de datos access diseñada para el estudio. Las variables cuantitativas se resumieron con el valor mínimo, máximo, media y desviación típica. Las variables cualitativas se resumieron mediante distribución de frecuencias e intervalo de confianza del 95\%. Con la finalidad de comparar las respuestas obtenidas en la encuesta en función de la variable categoría profesional, se empleó la prueba de $\mathrm{chi}^{2}$. El análisis de los datos se realizó mediante el programa SPSS 14.0 para Windows.

\section{RESULTADOS}

La encuesta se distribuyó a 793 profesionales de atención primaria del área sanitaria 8 de Madrid. Se hizo llegar a los centros a través de la gerencia de area, los/as técnicos/as de salud y de los/as responsables de enfermería. Dentro de ellos se distribuyó a los/as profesionales a través del referente de violencia de cada centro. La tasa de respuesta fue del 21,4\%. La distribución por variables sociodemográficas de los/as profesionales encuestados se muestra en las tablas 1 y 2 .

Respecto al lugar de trabajo el $81,1 \%$ trabajaban en centro de salud; $8,1 \%$ en la gerencia; $6,8 \%$ en el servicio de asistencia rural (SAR) y $4,1 \%$ en consultorio local.

Habían recibido formación el 35,7\% de los/as profesionales. La media de horas fue de 7,66 (DE 11,270), rango: 0-60. Por categoría profesional hubo $41,1 \%$ médi$\cos /$ as; $37,5 \%$ enfermeros/as; $12,5 \%$ auxiliares administrativos/as; 3,6\% trabajadores/as sociales, y $1,8 \%$ matrones/as, TCAE y celadores/as. 
Tabla 1

Distribución por variables sociodemográficas cuantitativasde los profesionales encuestados

\begin{tabular}{|l|c|c|c|c|}
\hline & Media & DE & Mínimo & Máximo \\
\hline Edad & 43,37 & 8,919 & 18 & 67 \\
\hline $\mathrm{N}^{\text {o de casos vistos }}$ & 0,88 & 1,46 & 0 & 10 \\
\hline
\end{tabular}

Tabla 2

Distribución por variables sociodemográficas cualitativas de los profesionales encuestados

\begin{tabular}{|c|c|c|}
\hline & Frecuencia & Porcentaje \\
\hline \multicolumn{3}{|c|}{ Sexo } \\
\hline Hombre & 21 & $13,80 \%$ \\
\hline Mujer & 131 & $86,20 \%$ \\
\hline $\mathrm{N} / \mathrm{C}$ & 18 & $10,60 \%$ \\
\hline \multicolumn{3}{|l|}{ Estado Civil } \\
\hline Casados/as & 88 & $62,40 \%$ \\
\hline Solteros/as & 40 & $28,40 \%$ \\
\hline $\begin{array}{l}\text { Separados/as } \\
\text { Divorciados/as }\end{array}$ & 11 & $7,80 \%$ \\
\hline Otros & 2 & $1,40 \%$ \\
\hline $\mathrm{N} / \mathrm{C}$ & 29 & $17,10 \%$ \\
\hline \multicolumn{3}{|l|}{ Categoría Profesional } \\
\hline Enfermeras/os & 55 & $34,40 \%$ \\
\hline TCAE & 2 & $1,30 \%$ \\
\hline Médicos/as & 58 & $36,30 \%$ \\
\hline Matronas & 3 & $1,90 \%$ \\
\hline Auxiliar Administrativo/a & 38 & $23,80 \%$ \\
\hline T. Social & 2 & $1,30 \%$ \\
\hline Celador/a & 2 & $1,30 \%$ \\
\hline $\mathrm{N} / \mathrm{C}$ & 10 & $5,90 \%$ \\
\hline
\end{tabular}

E: desviación estándar. $\mathrm{N}=170$

El $90,6 \%$ de los/as profesionales en los últimos 3 meses de su jornada laboral habían visto una media de 0,88 casos (DE 1,46). Mínimo 0 y máximo 10.

Los datos de las preguntas que se refieren al nivel de conocimientos que tienen los/as profesionales encuestados respecto a la VPHM, analizados por categoría profesional, se muestran en la tabla 3. Siendo los más relevantes: el $91,7 \%$ de los/as profesionales encuestados creía que las mujeres que padecen esta situación pasan desapercibidas en su 


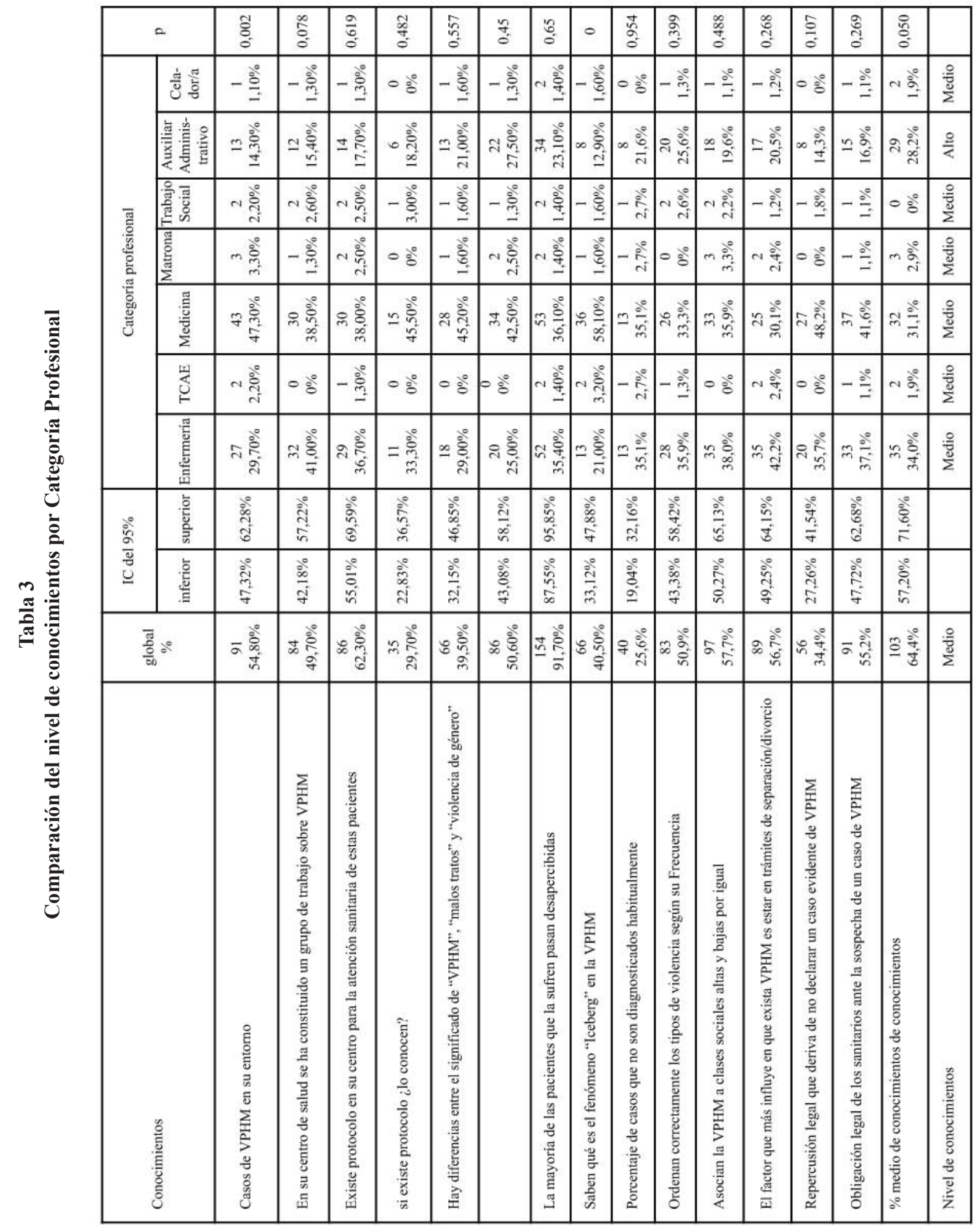


labor asistencial habitual. El 76,1\% afirman que la VPHM es una situación que afecta por igual a todo tipo de mujer, aunque hubo un pequeño porcentaje que la relacionaba con mujeres más vulnerables y dependientes económicamente del marido: extranjeras $12,3 \%$; amas de casa $7,4 \%$; mujeres sin estudios $4,3 \%$. E1 $62,3 \%$ sabían que existía un protocolo de actuación para la atención a estas mujeres en su centro, pero sólo lo conocía el $29,7 \%$. El 57,7\% no asociaba la violencia con clases sociales y el $56,7 \%$ creía que estar en trámite de separación o divorcio es un factor que influye en que la mujer reciba malos tratos por parte de su pareja o expareja. El 54,8\% conocía casos de VPHM en su entorno. El 50,6\% pensaba que es un problema muy frecuente o bastante frecuente, sin embargo, el $25,6 \%$ no conocía el porcentaje de casos no diagnosticados en los servicios sanitarios habitualmente.

El porcentaje de respuestas acertadas de forma global fue del $64,4 \%$. Alcanzaron un nivel de conocimientos de grado medio en todas las categorías profesionales, no encontrándose diferencias estadísticamente significativas entre ellas, excepto en el colectivo de trabajo social, cuyo nivel de conocimientos fue alto (tabla 3 , figura 1 ).

Respecto a las opiniones que tienen los/as profesionales acerca de la VPHM, se muestran los datos analizados por categoría profesional en la tabla 4. Los más relevantes fueron: para el 93,4\% la identificación de los casos de VPHM y la solución del problema debía de ser una labor multidisciplinar, competencia de policías, jueces/as, psicólogos/as, trabajadores/as sociales y a los propios sanitarios. Para el $87,2 \%$ el hombre que propicia la agresión contra la mujer es "como otro cualquiera". Para el 76,1\% afectaba a cualquier tipo de mujer. Para el 70,7\% este proble- ma es muy importante o bastante importante, pero sin embargo, el $69,3 \%$ ante una paciente con lesiones físicas, se planteaba la VPHM como un diagnóstico diferencial y el 55,0\% mantenían una actitud expectante habitualmente en su trabajo para detectar estos casos.

El 52,5\% consideraba necesario mejorar los sistemas de detección y la coordinación entre los distintos profesionales implicados en la atención integral a estas mujeres, y el 51,8\% creía que hay problemas organizativos o estructurales en el trabajo que impiden detectar estos casos.

Los/as profesionales de AP consideraban que sus principales funciones eran proporcionar la asistencia sanitaria que precise la mujer, detectar precozmente, dar asesoramiento, información, acompañar, apoyar emocionalmente, comunicar el caso a otros/as profesionales implicados, concienciar a la mujer de la situación, realizar parte de lesiones si es preciso y hacer un seguimiento del proceso. Para aumentar el grado de concienciación en el resto de sus compañeros/as lo harían con estos tres argumentos:

1. La importante repercusión que tiene este problema, no sólo sobre la salud de quien lo padece sino también a nivel social, laboral y cultural.

2. La alta prevalencia de esta problemática.

3. La accesibilidad y la confianza que tiene la mujer en los/as profesionales de AP para revelar su problema. Hace falta mayor implicación profesional.

Los problemas estructurales y organizativos que influían en la infradetección de casos para los/as profesionales encuestados fueron: el 52,5\% tenía miedo a represalias por parte del maltratador y sentía inseguridad por considerar que tie- 
Figura 1

Nivel de Conocimientos por categoría profesional

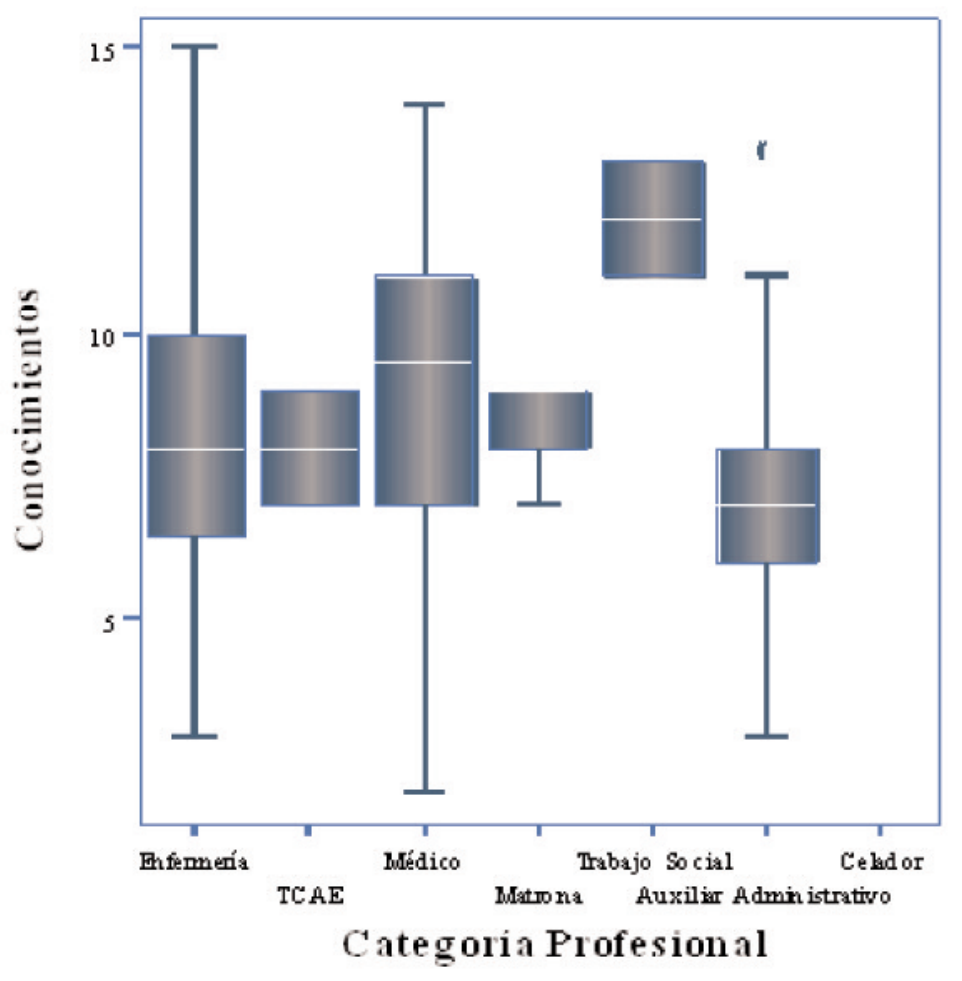

nen poco respaldo de otros/as profesionales y de la propia institución. El 29\% falta de tiempo para entrevistar a la paciente por la alta presión asistencial. El 23,5\% falta de formación al respecto. El 11,8\% desconocimiento del procedimiento a seguir en su centro. El 7\% desconocimiento de las competencias que tiene cada profesional. El 5,9\% falta de concienciación y de coordinación con otros/as profesionales implicados/as.

Para mejorar la detección de estas situaciones, si pudieran cambiar la organización del centro, planteaban: el 59\% formación específica; el 53\% disminuir la presión asistencial y aumentar el tiempo que puedan dedicar a este tipo de pacientes (actualmente 5 minutos en consulta médica y 10 minutos en consulta de enfermería); el 47\% realizar protocolos unificados y consensuados con criterios claros de actuación de cada profesional implicado; el 29,4\% poder garantizar confidencialidad a la mujer, escucha activa y empatía para que les cuente la problemática; el 5,9\% incluir la valoración de un/a psicólogo/a; el 4,7\% implicación de todos/as los miembros del equipo para garantizar una asistencia de calidad e integral a estas pacientes. 


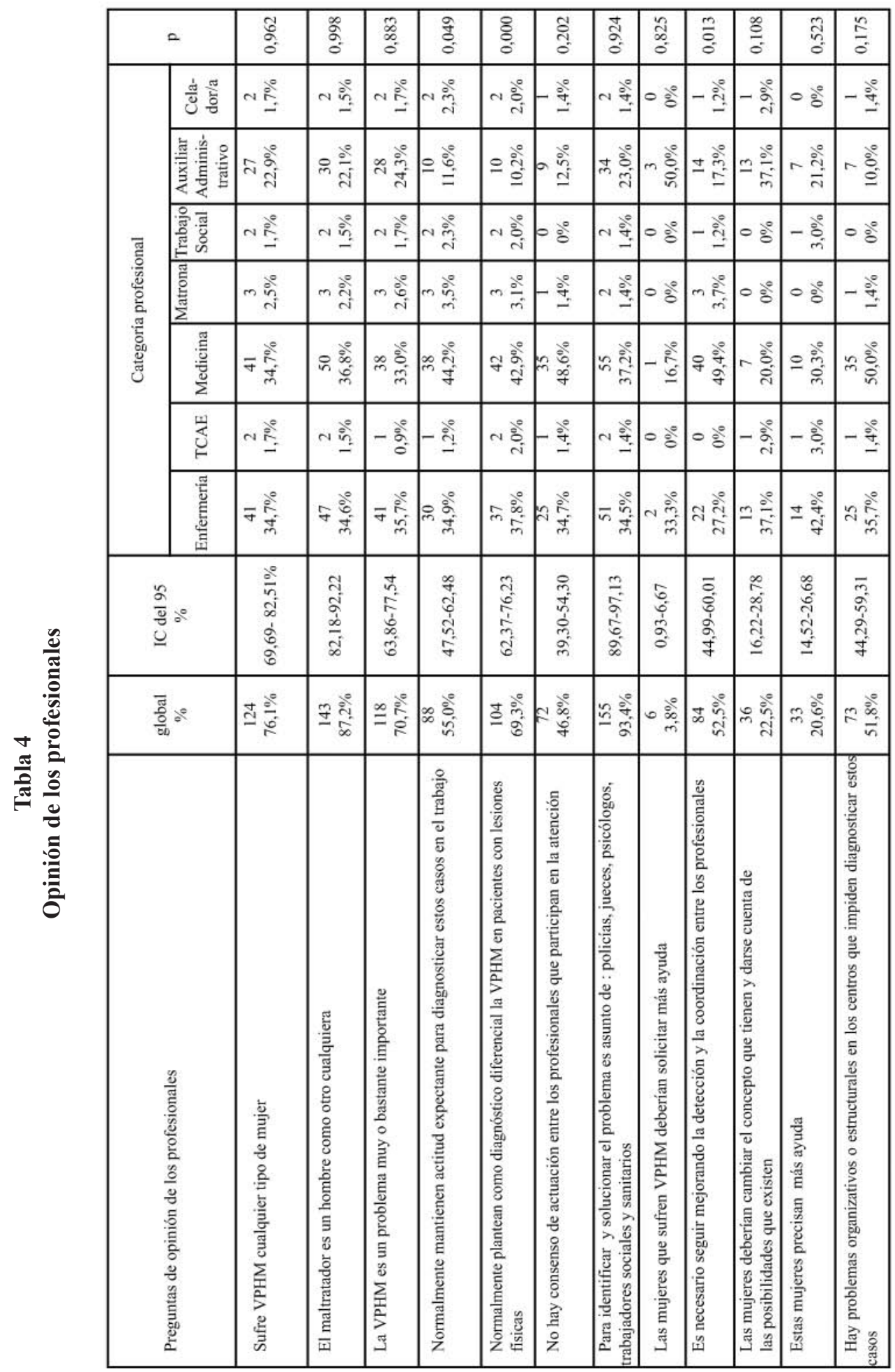

Rev Esp Salud Pública 2012, Vol. 86, N. ${ }^{\circ} 1$ 


\section{DISCUSIÓN}

Es importante señalar que en el estudio hemos encontrado tasas de participación mucho más bajas que en otros estudios previos $^{19-23}$. En ellos, las tasas participación están entre el 97,2\% (Nicaragua y México) y el $88,4 \%, 74 \%, 60 \%$ en los estudios realizados en hospitales de España. En nuestro estudio, dada la dispersión y amplitud del área, utilizamos los cauces habituales de distribución recomendados por la gerencia para que llegara a todos/as los/as profesionales, a diferencia de los anteriormente mencionados cuya distribución fue más personalizada.

Respondieron a la encuesta más mujeres, si bien es cierto que el porcentaje de ellas que trabajan en AP en esta área es superior al de hombres. Todos los colectivos habían recibido formación específica, sin embargo, son pocos los/as profesionales que declaran haber detectado casos en su jornada laboral.

A pesar de la elevada prevalencia y del impacto que tiene la VPHM en la salud de la mujer ${ }^{24}$, los/as profesionales sanitarios de AP siguen teniendo dificultades a la hora de detectar y abordar esta problemática.

El nivel de conocimientos es medio para todas las categorías profesionales estudiadas, excepto para los trabajadores sociales que es alto. La mitad de los/as profesionales saben que existe un protocolo de actuación en su centro, pero sólo lo conocen una tercera parte de ellos/as. Esto podría indicar desmotivación de los/as profesionales, falta de confianza en la propia competencia profesional y en la de otros/as profesionales implicados en la atención integral a las personas que padecen estas situaciones, como señalan los estudios anteriormente mencionados. Detectamos que existe un conocimiento general de la importancia de la VPHM en la salud, sin embargo, los/as profesionales no han adquirido todavía herramientas suficientes como para responder con solvencia ante los casos concretos.
Sí bien mas de la mitad de los/as profesionales encuestados/as consideran que la VPHM es un problema bastante o muy importante, que además es frecuente y que está presente en nuestro entorno, sin embargo, son muchos/as más los/as que declaran que pasa desapercibido, que no se plantean el diagnóstico diferencial incluso cuando la mujer acude a consulta con lesiones físicas y que han visto muy pocos casos en su práctica asistencial. Constatamos, por tanto, un contraste acentuado entre la prevalencia de VPHM, la asidua asistencia de estas pacientes a las consultas de AP por diferentes síntomas derivados de esta problemática, la concienciación de los/as profesionales respecto a la prevalencia de la violencia en la salud y la escasa cifra de casos reales detectados por ellos/as. En cuanto a la actitud expectante y la posibilidad de establecer un diagnóstico diferencial que haga posible la detección del problema, los resultados obtenidos en nuestro estudio son similares a los de otros estudios ${ }^{19-23}$. Menos de la mitad de los médicos/as se plantean la VPHM como diagnóstico diferencial, sin que por ello se hayan planteado cambios o propuestas efectivas.

Aproximadamente un tercio de los/as profesionales mantiene estereotipos respecto a la víctima y al maltratador. Según la bibliografía actual disponible, es posible que esto influya en la detección ${ }^{25-28}$. Parece interesante plantear despitajes básicos que implementen la detección, como recoge otro estudio consultado ${ }^{25} \mathrm{y}$, una vez detectados, se podrían manejar a través de un/a referente con formación específica al/a que podrían acudir el resto de los/as profesionales del equipo. Recientemente, en el área de estudio se ha creado la Comisión de Atención Primaria contra la violencia de género .

Es preocupante que más de la mitad de los/as profesionales encuestados desconozcan la repercusión legal que tiene el no comunicar los casos de violencia a otros/as profesionales implicados/as. Pero una vez 
detectado el caso, la mitad de los/as encuestados/as conocen la obligatoriedad que tienen de ponerlo en conocimiento de la autoridad competente. Esto refleja la complejidad existente en la relación entre la práctica clínica y la legislación vigente. La ley Orgánica 1/2004, de Medidas de Protección Integral contra la violencia de Género ${ }^{29}$ contempla, que, ante la mera sospecha, el profesional debe ponerlo en conocimiento de la autoridad competente. Así se contempla también en la Ley 5/2005, Integral contra la violencia de Género de la Comunidad de Madrid $^{30}$.

Los problemas que con más frecuencia se repiten son la falta de tiempo para indagar acerca de la violencia debido a la sobrecarga asistencial, el desconocimiento de los protocolos de actuación y de las competencias profesionales, la falta de formación específica, la falta de coordinación eficaz con otros/as profesionales. Estos mismos problemas aparecen en otros estudios consultados ${ }^{19,20,22,23}$.

Las propuestas de mejora más relevantes serían formación específica, disminución de la presión asistencial y realización protocolos unificados y consensuados que mejoren la coordinación de los profesionales.

En vista de los resultados, además de seguir desarrollando programas de formación específicos, parece necesario introducir otras estrategias formativas y organizativas que incidan en la adquisición de herramientas para la intervención de los casos concretos y en la implementación de la coordinación con otros profesionales implicados.

Como limitaciones del estudio, además de las propias del diseño transversal de la investigación, sería conveniente estudiar las causas de la baja tasa de respuestas que hemos obtenido, ya que introduce un sesgo, así como haber realizado una estimación de encuestas por categoría profesional que mejorara las comparaciones entre estas, evitando diferencias tan importantes en la tasa de respuesta. Consideramos que sería interesante ampliar la muestra a todos los centros de AP de la Comunidad de Madrid para conocer si existen diferencias estadísticamente significativas entre las distintas áreas sanitarias.

Como conclusiones destacamos que los/as profesionales de AP, pese a la formación recibida, siguen teniendo dificultades a la hora de detectar e intervenir en los casos de VPHM. La coordinación entre los servicios implicados en la atención integral a las mujeres que sufren estas situaciones debe implementarse, ya que la complejidad del problema hace que deba abordarse desde un punto de vista multidisciplinar. Plantear una formación conjunta y unos protocolos de actuación complementarios y coordinados podrían garantizar más seguridad a la mujer y una atención de calidad. Sería recomendable promover registros que recojan información unificada que nos permitan conocer la situación real de esta problemática en AP en la comunidad de Madrid y proponer nuevas investigaciones en este campo.

\section{BIBLIOGRAFÍA}

1. La violencia doméstica contra mujeres y niñas. Fondo de las Naciones Unidas para la Infancia. Florencia: Centro de investigaciones Innocenti; 2000. Documento Innocenti Digest núm.6.

2. Declaración sobre la eliminación de la violencia contra la mujer. Asamblea General de la Naciones Unidas; 1993. A/RES/48/104.23 de febrero de 1994. Disponible en:

http://www.acnur.org/biblioteca/pdf/1286..pdf

3. La eliminación de la violencia contra la mujer. Resolución de la Comisión de Derechos Humanos de las Naciones Unidas; 2005/41 del 19 de abril de 2005. Disponible en:

http://www.acnur.org/biblioteca/pdf/3447.pdf

4. World Health Assambly. Resolución WHA 49.25. 49a Asamblea Mundial de la Salud. OMS; 1996. Disponible en:

http://www.who.int/violence injury prevention/resources/publications/en/WHA4925_spa.pdf. 
5. Informe Mundial Sobre la Violencia y la Salud. Organización Mundial de la Salud; 2002. Disponible en: h t t p : / / w h libdoc. who.int/publi c a tions/2002/9275324220_spa.pdf

6. Estudio Multipaís sobre Salud de la Mujer y Violencia Doméstica. Organización Mundial de la Salud. Departamento de género mujer y salud. Ginebra 2005: 1, 7-11. Disponible en:

http://www.who.int/gender/violence/who_multicountry_s tudy/summary_report/summaryreportSpanishlow.pdf .

7. III Macroencuesta sobre la Violencia Contra las Mujeres. Instituto de la Mujer; abril 2006. Disponible en: http://www.madrid.org/cs/Satellite?blobcol=urldata\&blo bheader=application/pdf\&blobkey=id\&blobtable=MungoBlobs\&blobwhere $=1220373748592 \&$ ssbinary $=$ true

8. Informe sobre víctimas mortales de la violencia de género y de la violencia doméstica en el ámbito de la pareja o expareja en 2010. Consejo General del Poder Judicial. Sección del Observatorio contra la violencia doméstica y de género. Disponible en: http://www.observatorioviolencia.org/upload_images/File/DOC1306844610_victimas_2010.pdf

9. Datos Estadísticos Judiciales en aplicación de la L.O. 1/2004 Observatorio Contra la Violencia Doméstica y de Género 2010 . Disponible en: http://www.poderjudicial.es/eversuite/GetDoc?DBNa$\mathrm{me}=\mathrm{dPortal} \&$ UniqueKeyValue $=156089 \&$ Download $=$ fal se\&ShowPath=false

10. Barómetro Sanitario del Centro de Investigaciones Sociológicas; 2009 . Disponible en: http://www.msps.es/estadEstudios/estadisticas/docs/BS 2009_Presentacion.pdf

11. Guía de Apoyo en Atención Primaria de la Comunidad de Madrid para abordar la Violencia de Pareja Hacia la Mujer. Disponible en:

http://www.madrid.org/cs/Satellite?blobcol=urldata\&blo bheader=application $\% 2$ Fpdf\&blobheadername $1=$ Contentdisposition \&blobheadername $2=$ cadena\&blobheadervalue $1=$ filename $\% 3$ DGUIAVPM.pdf\&blobheadervalue $2=$ language $\% 3$ Des $\% 26$ site $\% 3$ DPortalSalud\&blobkey $=$ id \&blobtable $=$ MungoBlobs \&blobwhe$\mathrm{re}=1202765810355 \&$ ssbinary $=$ true

12. Coll Vinent B, Echevarria T, Farrás U, Rodríguez D, Millá J, Santiñá M. El personal sanitario no percibe la violencia doméstica como un problema de salud. Gac. Sanit. 2008;22:7-10

13. Ruiz Pérez I, Plazaola Castaño J, Del Río Lozano M. Physical health consequences of intimate partner violence in Spanish women. Eur J Public Health. 2007; 17:437-43.
14. Hegarty KL, Bush R. Prevalence and associations of partner abuse in women attending general practice: a cross sectional survey. Aust NZ J Public Health. 2002 oct; 26(5): 437-42. Disponible en: http://www.ncbi.nlm.nih.gov/publimed/12413288

15. Alice Kramer, RN, MS Darcy Lorenzon, MS and George Mueller, PhD. Prevalence of intimate partner violence and health implications for women using emergency department and primary care clinics. Wisconsin 2003 dec; Elsevier. Women's Health Issues 14(2004): 19-29. Disponible en: http://www.nnvawi.org/.../Kramer_partner_violence_health_implications.pdf.

16. Ruiz Pérez I, Plazaola Castaño J, Blanco Prieto P, González Barranco JM, Ayuso Martín P, Montero Piñar MI, Grupo de Estudio para la Violencia de Género. La violencia contra la mujer en la pareja: un estudio en el ámbito de la atención primaria. Barcelona 2006. Gac Sani.t20(3):202-8.Disponible en: http://scielo.isciii.es/scielo.php?pid=S021391112006000300006\&script $=$ sci_arttext

17. Feder G, Ramsay J, Dunne D, Rose M, Arsene C, Norman R, Kuntze S, Spencer A, Bacchus L, Hague G, Warburton A, and Taket A. How far does screening women for domestic (partner) violence in different health-care settings meet criteria for a screening programme?. Systematic reviews of nine UK National Screening Committee criteria. UK 2009. Health Technology Assesment 13 (16): abstract. Disponible en: http://www.hta.ac.uk/fullmono/mon1316.pdf

18.Ruiz Pérez I, Plazaola Castaño J, Vives Cases C, Montero Piñar MI, Escribà Agüirb V, Jiménez Gutiérrez E, Martín Baena D, y G6 para el Estudio de la Violencia de Género en España. Variabilidad geográfica de la violencia contra las mujeres en España. Barcelona 2010; gaceta sanitaria 24(2). Disponible en:

http://www.ucm.es/info/seas/comision/tep/mt/Vari abilidad_geografica_de_la_violencia_contra_las_ mujeres\%20en_Espana.pdf

19. Siendones Castillo R, Perea-Milla López E, Arjona Huertas JL, Aguera Urbano C, Rubio Gallo A, Molina Molina M. Violencia doméstica y profesionales sanitarios: conocimientos, opiniones y barreras para la infradetección. Emergencias 2002; 14 (5):224-232.

20.Arredondo Provecho AB, Del Pliego Pilo G, Nadal Rubio M, Roy Rodríguez R. Conocimientos y opiniones de los profesionales de la salud de atención especializada acerca de la violencia de género. Enferm Clín. 2008;18(4):175-82 
21.Larrauri Barandela MA, Rodríguez Alonso E, Castro Feijoó TM, Alfonso Pérez Z, Ojea Montes B, Blanco Gómez MA. Conocimientos y actitudes de los profesionales de urgencias sobre violencia de género. Enf urg. 2010; Vol II:36-39.

22. Rodríguez Bolanos R A, Márquez Serrano M Kageyama Escobar M L. Violencia de género: actitud y conocimiento del personal de salud de Nicaragua. Salud Pública Méx. 2005; 47(2): 134-144.

23. Méndez Hernández P, Valdez Santiago R, Viniegra Velázquez L, Rivera Rivera I, Salmerón Castro J. Violencia contra la mujer: conocimiento y actitud del personal médico del Instituto Mexicano del Seguro Social, Morelos, México. Salud Pública Méx. 2003; 45 (6): 472-482.

24. Magnitud, impacto en salud y aproximación a los costes sanitarios de la violencia de pareja hacia las mujeres en la Comunidad de Madrid: Comunidad de Madrid; 2008. Documentos Técnicos de salud Pública.

25. Pueyo J. La actitud del personal sanitario ante el maltrato contra las mujeres. Primeras Jornadas sobre violencia intrafamiliar. Universidad de Zaragoza; 2002. Disponible en: http//www.unizar.es/sociología_juridica/viointrafamiliar/pueyo.pdf

26. Freund KM, Ba KSM, Blackhall L. Identifying domestic violence in primary care practice. Evans department of clinical research, Boston University Clinical Center. J Gen intern Med 1996 Jan; 11(1) 44-6 Abstract.

27.García Torrecillas J M, Torío Durántez J, Lea Pereira M C, García Tirado M C, Aguilera Tejero R. Detección de la violencia contra la mujer en la consulta del médico de familia. Aten Primaria. 2008; 40:455-61. Vol.40 núm. 09. Disponible en:

www.elsevier.es/en/node/2067650

28.Gregory A, Ramsay J, Agnew-Davies R, Baird K, Devine A, Dunne D, Eldridge S, Howell A, Johnson M, Rutterford C, Sharp D, Feder G. Primary care Identification and Referral to Improve Safety of women experiencing domestic violence (IRIS): protocol for a pragmatic cluster randomized controlled trial. Disponible en: www.biomedcentral.com/14712458/10/54/abstract.

29.Ley Orgánica 1/2004, de 28 de diciembre, de Medidas de Protección Integral contra la Violencia de Género. $\mathrm{BOE} \mathrm{n}^{\circ} 313$ (29 de diciembre 2004).

30.Ley $5 / 2005$, de 20 de diciembre, Integral contra la violencia de Género de la Comunidad de Madrid. $\mathrm{BOCM} \mathrm{n}^{\circ} 310$ (29 de diciembre de 2005). 


\section{Anexo 1 \\ Encuesta a Profesionales Sobre Violencia de Pareja Hacia la Mujer}

Modificada de la utilizada en el estudio de Siendones Castillo R, et al. Violencia doméstica y profesionales sanitarios (19).

1. ¿Conoces algún caso de violencia de pareja hacia la mujer en tu entorno? $\square$ Sí $\square \square \square \square \square \square \square$ NO

2. ¿Cuántos casos habrás visto en los últimos 3 meses durante tu jornada laboral? $\quad N^{\circ} \ldots \ldots \ldots . . . .$.

3. ¿Sabias que en tu centro de salud se ha constituido un grupo de trabajo sobre violencia de pareja hacia la mujer?

$\square$ Sí, ya lo sabía $\square \square$ No, es la primera noticia que tengo del tema

4. ¿Existe protocolo interno en tu centro de salud para la atención sanitaria de estas pacientes? SÍ $\square \square \square \square$ NO

4.1. En caso de existir, ¿lo conoces? Sí $\square \square \square \square$ NO

5. ¿Crees que el significado de "violencia de pareja hacia la mujer"; "Malos Tratos"; "Violencia de género" es el mismo o crees que hay diferencias entre ellos? $\quad \square$ Son similares los tres $\square$ Hay diferencias $\square$ (explicalo)

6. Piensas que la violencia de pareja hacia la mujer en nuestra sociedad es un problema:

$\square \square \square \square \square \square \square \square$ Muy frecuente $\quad$ Bastante frecuente $\quad$ Algo frecuente $\quad$ Poco frecuente

7. Crees que la mayoria de las pacientes que la sufren: $\square$ Comunican su situación $\square$ Pasan desapercibidas

8. ¿Sabes lo que es el fenómeno "Iceberg" en la violencia de pareja hacia la mujer? $\square$ NO $\square$ SI $\square \square$ explicalo)

9. ¿Conoces cuál podría ser el porcentaje de casos que NO son diagnosticados habitualmente?

$10-30 \% \quad 30-50 \% \quad 50-70 \% \quad 70-90 \%$

10. ¿Sabrias ordenar de más a menos frecuente los tipos de violencia de pareja hacia la mujer? (un 1 para el más frecuente y un 3 para el menos frecuente): $\square$ Físico Psicológico $\square$ Sexual

11. Se asocia más la violencia de pareja hacia la mujer a clases sociales $\square$ Altas $\square$ Bajas $\square$ Las dos por igual

12. Según tu opinión, las mujeres que sufren violencia de pareja hacia la mujer con más frecuencia son:

. Amas de casa $\square$ Mujeres sin estudios $\square \quad$ Extranjeras Cualquier tipo de mujer

13. Habitualmente el maltratador es un hombre:

$\square$ Bebedor $\square$ Consumidor de drogas $\square \quad$ En paro "como otro cualquiera".

14. ¿Cuál de estos tres factores crees que influye más en que exista violencia de pareja hacia la mujer en nuestro medio? $\square$ Nivel sociocultural bajo $\square$ Estar en trámites de separación /Divorcio $\square$ Nivel socioeconómico bajo

15. ¿Conoces las repercusiones legales que puede derivar de no declarar un caso evidente de violencia de pareja

hacia la mujer? $\square$ Sí $\square$ NO

16. ¿Conoces las obligaciones legales de los sanitarios ante la mera sospecha de un caso de violencia de pareja hacia la mujer?

$\square$ Sí $\square$ NO

17. ¿Crees que es importante el problema de la violencia de pareja hacia la mujer?

$\square$ Muy importante $\square$ Bastante importante $\square$ Algo importante $\square$ Poco importante

18. En tu trabajo habitual, ¿mantienes una actitud expectante para diagnosticar estos casos?

$\square$ Siempre Si $\square$ Normalmente Si $\square$ Normalmente NO $\square$ Siempre NO

19. En tu trabajo, ante una paciente con lesiones fisicas, ¿te planteas como diagnóstico diferencial la posibilidad de que sea un caso de violencia de pareja hacia la mujer?
$\square$ Siempre Sí
जormalmente Sí
$\square$ Normalmente NO
$\square$ Siempre NO

20. ¿Crees que hay consenso en la actuación de todos los profesionales que participan en la atención de la mujer victima de violencia de pareja?

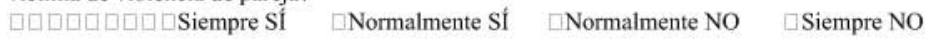

21. A la hora de identificar y solucionar este problema, crees que es un asunto más de la/los

$\square \square \square \square$ policia $\square$ jueces $\square$ psicólogos $\square$ trabajadores sociales $\square$ sanitarios $\square$ todos

22. ¿Cuál de estas opiniones crees que se adapta más a la situación actual de este problema, en relación con la infradetección?. (Elegir una opción)

A: $\square$ Creo que las pacientes que sufren violencia de pareja deberian insistir más, tener una postura más firme y solicitar más la ayuda de la sociedad. 
B: Creo que es necesario seguir mejorando los sistemas de detección y la coordinación entre los distintos estamentos y profesi onales implicados.

C: Estas pacientes piensan que su problema no tiene solución y que la sociedad no las respalda. Creo que ellas necesitarian cambiar este concepto erróneo y deberian darse cuenta de las posibilidades que existen.

D: Creo que las pacientes que sufren violencia de pareja no disponen todavia de las facilidades necesarias y precisan más ayuda.3. ¿Cuáles crees que son las principales funciones de los sanitarios y las urgencias en este problema?

23. ¿Cuáles crees que son las principales funciones de los sanitarios y las urgencias en este problema?

24. Si quisieras modificar el comportamiento de los sanitarios, aumentando su grado de concienciación. ¿Con qué 3 razonamientos lo harias?

25. ¿Crees que hay problemas organizativos o estructurales en tu trabajo que impiden diagnosticar estos casos?

$\square$ No $\quad \square$ Si $\square$ ¿Por qué?

26. Si pudieras cambiar el organización funcional de tu lugar de trabajo. ¿Qué harías o cambiarías para mejorar la capacidad de detección de mujeres que sufren violencia de pareja?

27. Categoría Profesional a la que perteneces: $\square$ Enfermería $\square$ Auxiliar de Enfermeria $\square$ Médico/a $\square$ Matrona $\square$ Fisioterapeuta $\square$ Trabajo Social $\square$ Celador/a $\square$ Aux. Administrativo/a

28. Desempeñas tus funciones en: $\square$ Centro de Salud $\square$ Consultorio Local $\square$ SAR $\square$ Gerencia

29. Sexo: $\square$ Hombre $\square$ Mujer

30. Edad:

31. Estado civil: $\square \square$ Casado/a $\square$ Soltero/a $\square$ Viudo/a $\square$ Separado/a-divorciado/a $\square$ Otros

32. ¿Has realizado algún curso de formación sobre violencia de pareja hacia la mujer? $\quad \square$ No $\quad \square \mathrm{Si}$

33. ¿De cuántas horas aproximadamente?..........

Los datos de la encuesta se contestan de forma anónima, pero sus resultados se podrán difundir públicamente.

Muchas gracias por su participación 